\title{
Effects of medical scribes on physician productivity in a Canadian emergency department: a pilot study
}

\author{
Peter S. Graves MD, Stephen R. Graves BSc, Tanvir Minhas BSc, Rebecca E. Lewinson BSc, \\ Isabelle A. Vallerand PhD, Ryan T. Lewinson MD PhD
}

\section{Abstract}

Background: Emergency department efficiency is a priority across Canada. In the United States, scribes may increase the number of patients seen per hour per physician; however, Canadian data are lacking. We sought to implement scribes in a Canadian emergency department with the hypothesis that scribes would increase the number of patients seen per hour per physician.

Methods: We conducted a 4-month quality improvement pilot study in a community emergency department in Ottawa, Ontario. Data collection began January 2015 after scribe training. Physicians received shifts with and without a scribe for a period of 4 months. Across the study, the mean number of patients seen per hour was determined for each physician during shifts with and without a scribe. We compared mean ( \pm standard deviation $[S D]$ ) number of patients seen per hour based on presence or absence of a scribe by 2-tailed paired-samples $t$ test.

Results: Eleven scribes participated and ranged in age from 18 to 23 years. Twenty-two full- or part-time emergency physicians were followed. We documented 463 physician-hours without use of a scribe and 693.75 physician-hours with use of a scribe. Across all 22 physicians, $18(81.8 \%)$ saw more patients per hour with use of a scribe. Overall, the number of patients seen per hour per physician was significantly greater $(+12.9 \%)$ during shifts with a scribe (mean $[ \pm \mathrm{SD}] 2.81[ \pm 0.78]$ ) than during shifts without a scribe (mean $[ \pm \mathrm{SD}] 2.49[ \pm 0.60] ; p=0.006)$.

Interpretation: In this pilot study, the use of scribes resulted in an increased number of patients seen per hour per physician. Because this was a small study at a single centre, further research on the effects of scribes in Canada is warranted.

I mproving emergency department efficiency and productivity has been a priority in hospitals across Canada, and there is a need for cost-effective, evidence-based solutions to address this concern. In addition, electronic medical records are becoming common, and have the advantage of improving continuity of care through better documentation; however, they may have a negative impact on productivity for some physicians. ${ }^{1}$

Medical scribes provide real-time medical transcription of the physician-patient encounter, flow management and clerical support. Their use has been shown to improve the physician-patient interaction, increase physician productivity and facilitate better continuity of care owing to a more accurate, complete and legible medical record. ${ }^{2-4}$ Overall, these potential benefits may lead to reduced hospital costs. ${ }^{5}$ However, these data are largely based on research from the United States. Despite differences between the American and Canadian health care systems, we believe the benefits of having medical scribes may be transferable. Unfortunately, very limited data exist on the use of medical scribes in Canadian emergency departments. In this study, we assessed the effect of introduc-

Competing interests: Peter Graves and Stephen Graves are owners of Medical Scribes of Canada. Tanvir Minhas and Rebecca Lewinson were employees of Medical Scribes of Canada at the time of the study. Peter Graves, Stephen Graves and Tanvir Minhas report funding from the National Research Council Industrial Research Assistance Program. Peter Graves and Stephen Graves report funding from the QueenswayCarleton Hospital. No other competing interests were declared.

This article has been peer reviewed.

Correspondence to: Peter Graves, pgraves@mediscribecanada.ca CMAJ Open 2018. DOI:10.9778/cmajo.20180031 
ing medical scribes in a Canadian community hospital emergency department. The objectives of this pilot study were to establish proof-of-concept of medical scribes in Canada and to gain experience in scribe implementation so as to inform the development of larger multicentre trials. Based on data from the United States, we hypothesized that using a medical scribe would be associated with greater physician productivity.

\section{Methods}

\section{Setting}

We conducted a 4-month community-based quality improvement pilot study of the use of medical scribes in the emergency department at the Queensway-Carleton Hospital in Ottawa, Ontario, from January 2015 to April 2015. The hospital is a nonacademic community hospital with 24 full- or part-time emergency physicians (at the time of data collection) and about 70000 annual emergency department patient visits.

\section{Scribe training and implementation}

Medical scribes provide real-time medical transcription of the physician-patient encounter, flow management and clerical support. Medical scribes are trained in medical terminology and confidentiality, and are held to the same standards of confidentiality as a physician or nurse. After obtaining patient consent to have a scribe present, the scribe enters the room with the physician and is present during the patient-physician encounter. During the encounter, the scribe documents the history given by the patient, as well as the results of the physical examination, which are verbally stated by the physician to the scribe. After the encounter, the physician reviews the notes taken by the scribe to ensure that relevant and correct information is present before signing off on the chart. Outside of the room, the scribe helps manage the physician's flow by alerting them to patients who need to undergo reassessment or when new test results have returned.

The scribes who participanted in this study were supplied by Medical Scribes of Canada (Ottawa). Eleven scribes participated in the study who ranged in age from 18 to 23 years. As part of the Medical Scribes of Canada model, each scribe was paid on an hourly basis through a grant from the hospital. To recruit scribes, postings were made in the Ottawa area for undergraduate students enrolled in health-related degree programs. Applicants underwent screening based on their academic performance and work and volunteer experience before being formally interviewed by physicians and community members in a manner similar to medical school admissions. Thus, any differences in background or qualifications across scribes were kept to a minimum.

After being hired, all scribes underwent basic training in medical terminology and disease presentations commonly encountered in the emergency department. From November 2014 to December 2014, scribes were introduced to the emergency department at the hospital. Scribes gained experience and confidence in charting and patient interaction, and physicians became comfortable with using a scribe.

\section{Intervention}

After training and an initial 2-month acclimation period for both scribes and physicians, data collection began in January 2015. A convenience sample of 22 full- or part-time emergency physicians were followed. Physicians volunteered to receive shifts with and without a scribe for a period of 4 months. A total of 24 physicians were initially eligible to participate, but 2 declined to do so, citing their preference to work alone. All physicians were fully licensed for independent emergency medicine practice and either held certification in emergency medicine from the Royal College of Physicians and Surgeons of Canada or had received certification for special competence in emergency medicine from the College of Family Physicians of Canada. Scribe allocation to shifts was done by the Medical Scribes of Canada Human Resources Manager, who was blinded to all data collection and based scheduling primarily on scribe availability because many had full-time academic schedules. As a consequence, scribes were only allocated to evening shifts ( $4 \mathrm{pm}$ to $12 \mathrm{am} ; 6 \mathrm{pm}$ to $2 \mathrm{am}$ ), which are traditionally high volume. Effort was made to rotate scribes among physicians. Physician preferences to working with or without scribes, or for certain scribes, were not considered in allocation.

The emergency department is divided into a cubicles area, an observation area and a resuscitation bay. The cubicles area is used for more acute and localized cases such as fractures, lacerations, abscesses and acute abdominal pain. The observation area is used for complex cases, such as altered level of consciousness, respiratory concerns or cardiac monitoring, where the patient may be monitored for a long period. The resuscitation bay is used for major trauma, procedural sedation and critical care. In this study, the use of scribes was only evaluated in the cubicles area, because this tends to be where the hospital has the largest patient volumes. This setup helped control for any bias that could arise if certain physicians spent more of their shifts in the lower volume observation area. Because scribes were only allocated to evening shifts, only evening shifts were considered in nonscribe performance metrics.

Because we sought to evaluate the real-world implementation of scribes, who were contracted for a certain number of hours per week, more shifts had scribes than did not. However, we ensured that each physician worked shifts with and without scribes during the study period. Any shifts with or without scribes that had low patient volume were removed from analysis. Low patient volume was defined as more than 1 hour without new patients to see, excluding the final hour of a shift.

\section{Physician performance data and analysis}

Hospital records were used to extract physician work hours and numbers of patients seen by each physician during each shift to calculate patients per hour per physician for each shift. The mean number of patients seen per hour was determined for each physician during shifts with and without a scribe.

We used Stata version 14.2 I/C for data analysis. Significance was set at $p<0.05$. All analyses were performed 
by 2 blinded researchers. Two-tailed paired-sample $t$ tests were used to compare number of patients seen per hour within physicians based on presence or absence of a scribe. To determine whether the number of patients seen per hour during nonscribe shifts was biased because of ongoing scribe use during the study period, we performed a sensitivity analysis on available hospital data from January 2014 to April 2014, when no scribes were used. Metrics from 18 of the 22 physicians in the study were available for this period, and these values were compared with the 2015 metrics for shifts without a scribe using a 2-tailed pairedsample $t$ test.

In addition, we conducted a hypothetical cost-analysis, whereby the cost of using a scribe was contrasted with the revenue generated by the physician. This was based on typical physician billing codes and normal billings at our centre. Patient or physician feedback was not formally obtained as part of the study.

\section{Ethics approval}

Ethics approval for this study was obtained from the Queensway-Carleton Hospital. The manuscript was prepared in accordance with the SQUIRE statement for quality improvement studies. ${ }^{6}$

\section{Results}

Twenty-two emergency physicians (Table 1) volunteered to participate in this community-based study and had shifts both with and without a scribe during the 4-month study period. We documented a total of 463.0 physician-hours (61 shifts) without use of a scribe, and 693.75 physician-hours (97 shifts) with a scribe. Because this study included only 8 -hour evening shifts, physicians typically worked 2-3 shifts without a scribe and 4-6 shifts with a scribe during the course of the study. Only 1 shift without a scribe was deemed to be of low-patient volume shift and removed from consideration. No shifts with a scribe were low patient volume. Post-hoc analysis showed that the elimination of this single shift from consideration did not influence the final results.

\begin{tabular}{|lc|}
\hline \multicolumn{2}{|l|}{ Table 1: Physician characteristics } \\
\hline Characteristic & No. $(\%)^{\star}$ \\
\hline Sex & $12(55)$ \\
\hline Male & $10(45)$ \\
\hline Female & $9(41)$ \\
\hline Job type & $13(59)$ \\
\hline Full-time & $11.0( \pm 10.1)$ \\
\hline Part-time & \\
\hline Training & \\
\hline $\begin{array}{l}\text { Years with independent licence to practise, } \\
\text { mean }( \pm \text { SD) }\end{array}$ & \\
\hline $\begin{array}{l}\text { Note: SD }=\text { standard deviation. } \\
\text { *Unless otherwise indicated. }\end{array}$ \\
\hline
\end{tabular}

Patients seemed to only occasionally decline having a scribe present when their presenting concerns were sensitive in nature (e.g., mental health, genitalia-related). If the examination was sensitive in nature, the scribe stepped outside the room and the physician dictated the findings upon exiting. Informal feedback showed that patients found scribes to be a positive experience because the verbal dictation of findings to the scribe allowed physicians to explain what they were doing and why to the patients, in addition to what each finding meant in the specific clinical scenario.

Eighteen of the 22 physicians $(81.8 \%)$ showed a greater number of patients seen per hour with use of a scribe. Use of a scribe was associated with a significantly greater number of patients seen per hour per physician $(p=0.006$, mean increase of $12.9 \%$, range $-41 \%$ to $118 \%$ ); the mean number of patients seen per hour per physician over the duration of the study was 2.81 (stadndard deviation [SD] 0.78) with a scribe and 2.49 (SD 0.60) without (Figure 1).

In the sensitivity analysis, number of patients seen per hour per physician from no-scribe shifts did not differ between 2014 (mean $[ \pm$ SD $] 2.43[ \pm 0.43]$ ) and 2015 (mean $[ \pm$ SD] 2.53 $[ \pm 0.47])(p=0.3)$. The 2014 data were from evening cubicle shifts from January to April 2014.

Emergency physicians in Canada earn about $\$ 150 / \mathrm{h}$, equating to a net revenue of about $\$ 1200 /$ shift without a scribe (depending on province of practice, typical clinical loads, practice models and physician pace). At an hourly wage of $\$ 27$, a scribe would cost $\$ 216 /$ shift. Given that a scribe may be associated with a mean increase of about $13 \%$ in physician productivity, the hypothetical "cost" to a physician using a scribe would be about $\$ 60$ relative to what their earnings would be without a scribe. This cost may depend on the type of billing shift the physician is scheduled to, the usual pace of the physician and the volume in the emergency department. From a systems perspective, emergency physicians could possibly bill at a greater amount per shift due to increased volume of patient care. Based on our data, many physicians would

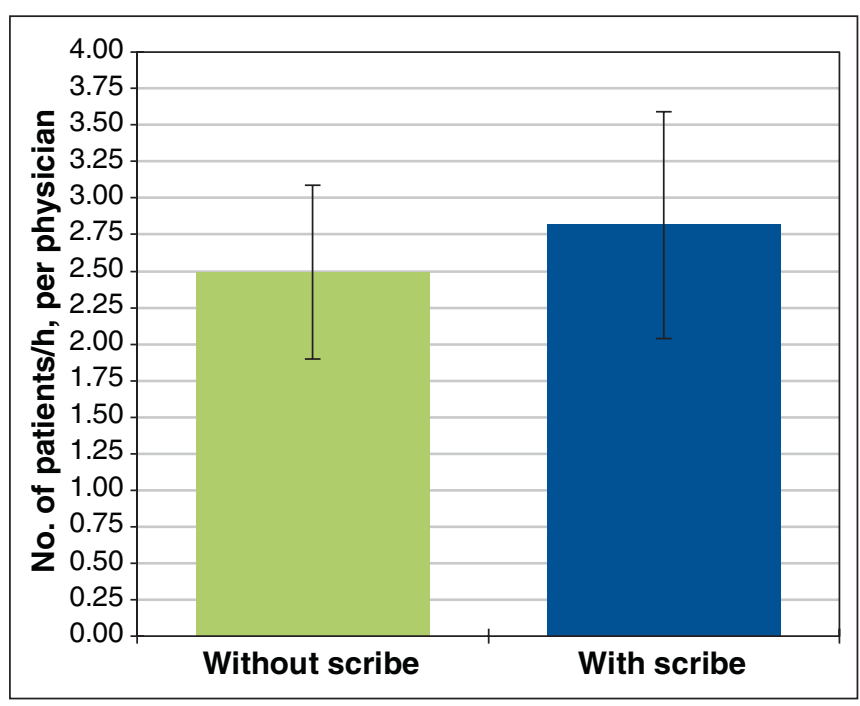

Figure 1: Physician efficiency with and without a scribe. These data represent mean values across all 22 physicians during the 4-month study. 
have earned a greater income even after accounting for scribe cost. Further research is needed to understand the full costbenefit implications of using scribes.

\section{Interpretation}

This pilot study assessed the effects of using medical scribes on physician productivity in a Canadian community hospital emergency department and provided insight toward developing a larger trial. Results showed that physician productivity was significantly higher with use of a scribe than without, which is in agreement with American data. Caution must be taken in extrapolating these findings to other Canadian centres, but the results are promising and suggest a need for further study. Currently, we have expanded the program to provide scribe services in a variety of centres and clinical settings to 25 physicians, including emergency physicians, orthopedic surgeons, urologists, oncologists and otolaryngologists.

In the US, use of scribes may contribute to increased number of patients seen per hour, ${ }^{3}$ improved physician and patient satisfaction $^{2}$ and increased time spent in patient interaction with decreased time spent reporting. ${ }^{7}$ Furthermore, productivity measures appear to be maintained for up to 1 year after implementation. ${ }^{8}$ In contrast to these findings, a previous Canadian study did not find a significant increase in the number of patients seen per hour with a scribe, ${ }^{9}$ although improved physician-nurse satisfaction and improved chart legibility were reported. This study took place in an academic hospital and did not indicate whether scribes received prestudy training. Such factors could have affected overall efficiency, because understanding content and documentation procedures may be critical to scribe performance. Moreover, it is possible that efficiency at large academic centres may be less affected by scribes owing to the tendency for more urgent or severe cases to be seen, which may occupy more resources; ${ }^{10}$ in addition, more time may have been spent teaching, or more trainees may have been on service.

Our sensitivity analysis showed that implementation of scribes did not result in a decrease in performance in physician shifts without a scribe relative to the previous year. Thus, the increased number of patients seen per hour metric in this study is a true increase related to the presence of scribes. However, it is not possible to discern the mechanism by which scribes contributed to the greater number of patients seen, be it through improved documentation, facilitated workflow or changes in physician behaviour. These aspects should be explored in further detail in larger studies.

Although we saw an overall increase in patients seen per hour per physician with scribes, some physicians $(n=4) \mathrm{did}$ not show this increase. Moreover, we found variability in the extent to which the patients seen per hour metric increased for each physician. Each physician likely has their own natural pace for seeing patients, and introducing a scribe may not produce the same size of effect for each physician. Thus, future studies should examine what factors predict beneficial effects versus inhibitory effects so that inhibitory factors can be directly addressed and remedied.

\section{Limitations}

This study was relatively small, with a short follow-up, at a single community hospital. Although our results suggested improved physician productivity with scribes, longer-term studies at multiple centres with more physicians are needed to assess generalizability.

We had informal conversations with patients and physicians regarding scribes, but this feedback was not measured as part of the study.

Some of the authors of this manuscript are the founders of Medical Scribes of Canada, and are employed at the Queensway-Carleton Hospital. Consequently, our ability to gain subjective data from emergency physicians on their experience with a scribe was affected, because it was felt that physicians could be biased in favour of their colleagues' business.

Patient characteristics, patient preferences and health -outcomes were not considered in this pilot study. Although clinical presentations were not likely to differ substantially between shifts with or without scribes, this possibility cannot be excluded.

Future studies would benefit from a multicentre design to identify whether scribe performance can be successful elsewhere. However, to do so, several technical challenges must be overcome. For example, different centres may use different health record systems. Because our scribes were incorporated into the health care team, they were given access to health records much the same way that medical students or residents would be. When implementing scribes into multiple centres, they would need access to and training for various systems; routinely switching from one system to another could impede their performance.

We only investigated evening shifts in the high-acuity ambulatory "cubicles" of our emergency department. Future studies may benefit from exploring the effects of scribes during different shift times and in different areas.

Measurement of physician and patient preferences, patient characteristics and clinical outcomes are all important outcome measures beyond physician productivity that should be considered in future studies. An interesting observation we noted for possible consideration is "physician departure time." Many of our participants noted that with scribes, they were better able to leave the hospital on time, or up to an hour earlier than scheduled, which could have implications for physician burnout. Thus, future studies should not only consider patient satisfaction, experience and outcomes with scribes, but also study satisfaction from the physician's perspective.

We allocated scribes to physicians mostly based on scribe availability, which could introduce bias. In future studies, scribes should be randomly allocated; however this necessitates having scribes available to work every shift.

\section{Conclusion}

This pilot study of the use of medical scribes in a Canadian community hospital emergency department provides initial proof-of-concept that their use may increase physician productivity. These results warrant further investigation in larger multicentre controlled trials where the effects of scribes can 
be more broadly assessed while considering additional covariates and outcomes relevant to patients, physicians and health care systems. The consideration of physician preferences, physician time in hospital, patients seen per hour, emergency department wait time, physician characteristics and clinical presentations, shift time, patient acuity and clinical setting (e.g., emergency, outpatient, inpatient), and scribe cost should all be considered in further detail.

\section{References}

1. National physician survey (2007, 2010 and 2014). Mississauga (ON): National Physician Survey. Available: http://nationalphysiciansurvey.ca/surveys/ (accessed 2018 Aug. 12).

2. Bastani A, Shaqiri B, Palomba K, et al. An ED scribe program is able to improve throughput time and patient satisfaction. Am 7 Emerg Med 2014; 32:399-402.

3. Heaton HA, Castaneda-Guarderas A, Trotter ER, et al. Effect of scribes on patient throughput, revenue, and patient and provider satisfaction: a systematic review and meta-analysis. Am 7 Emerg Med 2016;34:2018-28.

4. Misra-Hebert AD, Amah L, Rabovsky A, et al. Medical scribes: How do their notes stack up? 7 Fam Pract 2016;65:155-9.

5. Kreamer J, Rosen B, Susie-Lattner D, et al. The economic impact of medical scribes in hospitals. Physician Leadersh $72015 ; 2: 38-41$.

6. Davidoff F, Batalden P, Stevens D, et al. SQUIRE development group. Publication guidelines for quality improvement studies in health care: evolution of the SQUIRE project. BM7 2009;338:a3152.

7. Hess JJ, Wallenstein J, Ackerman JD, et al. Scribe Impacts on Provider Experience, Operations, and Teaching in an Academic Emergency Medicine Practice. West 7 Emerg Med 2015;16:602-10.
8. Heaton HA, Nestler DM, Lohse CM, et al. Impact of scribes on emergency department patient throughput one year after implementation. Am 7 Emerg Med 2017;35:311-4.

9. Chen PW, Dowling S, Abernethy R, et al. Assessment of scribes in Calgary emergency departments. Can F Emerg Med 2012;14(1):S29.

10. Tanabe P, Gimbel R, Yarnold PR, et al. The Emergency Severity Index (version 3) 5-level triage system scores predict ED resource consumption. 7 Emerg Nurs 2004;30:22-9.

Affiliations: Department of Emergency Medicine (P. Graves), Queensway-Carleton Hospital; Faculty of Medicine (P. Graves, Minhas), University of Ottawa; Medical Scribes of Canada (P. Graves, S. Graves, Minhas, R.E. Lewinson), Ottawa, Ont.; Faculty of Health (R.E. Lewinson), York University, Toronto, Ont.; Cumming School of Medicine (Vallerand, R.T. Lewinson), University of Calgary, Calgary, Alta.

Contributors: Peter Graves and Stephen Graves conceived and designed the study. Tanvir Minhas and Rebecca Lewinson acquired the data for the study. Isabelle Vallerand and Ryan Lewinson analyzed all data for the study and drafted the manuscript. Peter Graves, Steven Graves, Tanvir Minhas and Rebecca Lewinson provided critical input to the manuscript for revision. All of the authors approved the final version of the manuscript and agreed to act as guarantors of the results.

Funding: Queensway-Carleton Hospital Research Grant, National Research Council of Canada (NRC) Industrial Research Assistance Program. The Queensway-Carleton Hospital and NRC had no role in conducting the study, analyzing the data or drafting the results.

Supplemental information: For reviewer comments and the original submission of this manuscript, please see www.cmajopen.ca/content/6/3/ E360/suppl/DC1. 